\title{
Linx
}

Revue des linguistes de l'université Paris X Nanterre

$58 \mid 2008$

Aspects de comme

\section{Ellipses dans les constructions comparatives en comme}

Marianne Desmets

\section{CpenEdition}

Journals

Édition électronique

URL : http://journals.openedition.org/linx/328

DOI : $10.4000 /$ linx.328

ISSN : 2118-9692

Éditeur

Presses universitaires de Paris Nanterre

Édition imprimée

Date de publication : 1 juin 2008

Pagination : 47-74

ISSN : 0246-8743

\section{Référence électronique}

Marianne Desmets, «Ellipses dans les constructions comparatives en comme », Linx [En ligne],

58 | 2008, mis en ligne le 16 février 2011, consulté le 20 avril 2019. URL : http://

journals.openedition.org/linx/328; DOI : 10.4000/linx.328

Département de Sciences du langage, Université Paris Ouest 


\title{
Ellipses dans les constructions comparatives en comme
}

\author{
Marianne Desmets
}

\section{Des séquences fragmentaires dans les comparatives en comme $e^{1}$}

Que les constructions comparatives soient sujettes aux ellipses, au même titre que les constructions coordonnées, est un fait bien admis. On pourrait s'attendre à ce que les formes et les propriétés des ellipses soient identiques quel que soit le contexte dans lequel elles apparaissent. Or, tel n'est pas le cas. Nous montrons dans cet article que les ellipses des comparatives en comme satisfont, comme attendu, les contraintes syntaxiques et sémantiques générales du phénomène, mais qu'elles présentent, en revanche, des propriétés particulières qui les distinguent, entre autres, des ellipses des constructions coordonnées.

La comparative en comme a une distribution similaire à celle d'un adverbe de manière en -ment (Moline 2001, Desmets 2001). Elle connaît deux grands emplois : soit elle porte sur un constituant d'une phrase matrice (qu'elle modifie ou dont elle est complément), soit elle porte sur l'ensemble de cette phrase (à la manière d'un adverbe de phrase). Dans ses emplois comparatifs ${ }^{2}$, comme introduit une séquence de forme

\footnotetext{
${ }^{1}$ Je tiens à remercier A. Abeillé, P. Amsili, K. Baschung, L. Roussaire et F. Mouret pour leurs précieuses remarques. Toute erreur restant mienne.

2 Toutes les comparatives en comme ne permettent pas l'ellipse du verbe tête. Ainsi, l'ajout reportif, ajout incident à la phrase matrice qui permet la mention d'un discours (cf. Desmets \& Roussarie 2001) requiert obligatoirement la présence du verbe de discours ou de " pensée » :
} 
phrastique ou une séquence de (un ou plusieurs) constituants ne formant pas syntaxiquement une phrase complète ; ce que l'on nommera une séquence fragmentaire. Dans ce dernier cas, cependant, il n'y a pas lieu de postuler l'existence d'un autre type de construction comparative en comme puisque distributions et interprétations sont identiques quelle que soit la forme des séquences réalisées.

\subsection{Distribution}

Les séquences fragmentaires ou phrastiques en comme connaissent la même distribution. Elles se rencontrent en fonction d'ajout modifieur de Adj/SA (1), de $\mathrm{V} / \mathrm{SV}$ (2), ou de complément de manière d'un V (3) :

(1) a. il était donc convenable qu'il y eût des canaux et des ruisseaux visibles pour apporter ces grâces à chacun en particulier, visibles comme avait été visible la personne de l'homme-dieu. DICT. DE THEOLOGIE CATH. T.14 / 1938 : 537

b. tu seras laide comme les quatorze péchés capitaux ! COLETTE.G / CLAUdine A L'ECOLE / 1900

(2) a. Mais Ladourd retombe sur sa chaise, secoue la tête comme le taureau qui compte ses banderilles, mugit son indignation. BAZIN.H / LA MORT DU PETIT CHEVAL / 1950 : 105 / XIII

b. ... brave homme, très serviable... C'est drôle, mais quand j'entends ce mot dans la bouche de l'un des nôtres, il sonne exactement comme le mot serviette et donne l'impression d'être aussi facile à jeter dans le sac à linge sale. BAZIN.H / LA MORT DU PETIT CHEVAL / $1950: 69$ / VII

(3) a. Il le traita comme il aurait fait de ses propres enfants.

b. 25 avril-J'apprends que les danois, dans les rues de leurs villes, se comportent exactement comme les voitures dans les rues de Paris. BLOY.L / JOURNAL T.1 / 1900 : 320 / MON JOURNAL attribut (5):

Elles peuvent également être ajout modifieurs (i.e., épithète) de N/SN (4), ou

(4) a. ... dans ce trou de province, avec une pimbêche comme est ma nouvelle maittresse, je n'ai pas à rêver de pareilles aubaines, ni espérer de semblables distractions... je ferai du ménage embêtant... de la couture qui $\mathrm{m}$ ' assomme... rien d'autre... ah ! MIRBEAU.O / JOURNAL D'UNE FEMME DE CHAMBRE / $1900: 54$ /

b. Je regrette la bague comme avait mon amie Maud. HAMP.P / MAREE FRAICHE VIN DE CHAMPAGNE / 1909 : 231 / VIN DE CHAMPAGNE 1909

(i) En reconnaissant Koupriane, les deux nihilistes pouvaient, comme l'avait dit le reporter, se croire découverts, et précipiter la catastrophe. LEROUX.G

(ii) Il n'était surtout pas question de porter le voile, ce «symbole de la répression patriarcale», comme elle le qualifie. Le Monde DipL.

Nous signalons également que les comparatives ajouts, quand elles sont antéposées à la phrase, recoivent de meilleurs jugements d'acceptabilité lorsqu'elles sont fragmentaires. C'est le cas des comparatives d'analogie en comme, dont l'interprétation peut parfois entrer en collision avec celle d'un ajout causal, qui lui ne connait pas de forme elliptique (phénomène également signalé dans Moline 2007) : Comme Paul va au foot le mercredi soir, Marie va au tennis tous les samedis.

Le même phénomène s'observe avec les comparatives scalaires incidentes, qui présentent des difficultés à être antéposées lorsqu'elles ne sont pas fragmentaires (cf. Amsili \& Desmets, 2008b) : Plus que des subventions aux cités, il faut donner du travail aux jeunes vs *Plus qu'il faut donner des subventions aux cités, il faut donner du travail aux jeunes. 
c. Avec des yeux comme les tiens, on ne prend pas de rubans bleus, ça fait grincer des dents. (exemple modifié, d'après COLETTE.G / CLAUDINE A L'ECOLE / 1900 : 255)

(5) a. Paul est bien comme était ton père au même âge : toujours à lambiner.

b. vous êtes comme Achille, qui s'emporte contre la gloire, et comme le père Malebranche, dont l'imagination brillante écrivait contre l'imagination. GUEHENNO.J / JEAN-JACQUES T.2/

Les comparatives ont toujours la possibilité d'être modifiées par un adverbe de manière (6a), ou d'être coordonnées à un autre ajout de manière (6b), et ce, quelle que soit leur forme :

(6) a. Il m'aime, celui-là, précisément comme le chacal aime la panthère : pour les charognes qu'elle lui abandonne. BAZIN.H / LA MORT DU PETIT CHEVAL / $1950: 257$ / XXXI

b. ... un petit bonnet de linge fin... par exemple, des dessous riches : ça oui... mais écoutez bien... signez-moi un engagement de trois mois... et je vous donne un trousseau d' amour, tout ce qu' il y a de mieux, et comme les soubrettes du théâtrefrançais n' en ont jamais eu... ça, je vous en réponds... MIRBEAU.O / JOURNAL D'UNE FEMME DE CHAMBRE / $1900: 293$ /

En fonction adverbe de phrase, on observe les deux types de séquence. Le cas d'un ajout à la phrase, sémantiquement parenthétique et prosodiquement incident (au sens de Bonami et al. 2004), est illustré en (7) par une comparative dite "d'analogie » (qui met en relation deux propositions); on note la mobilité dans la phrase caractéristique des adverbes de phrase :

(7) a. Mais je ne nie pas qu'ils voient, car, comme les yeux ordinaires sont faits pour la lumière et appréhendent chaque chose par l'enveloppe qu'elle lui fait, l'on dirait que les tiens sont sensibles à la nuit seule, reconnaissant tout par le noyau, par la forme de l'opacité intérieure. CLAUDEL.P / LA J.F. VIOLAINE 2.VERSION / 1901 : 613 / ACTE III

b. Alors que comme les moutons dans la bergerie, quand le matin vient, sentant l'herbe bonne à manger, bêlants, s'accumulent contre les portes, j'entends un peuple vivant élever la voix. CLAUDEL.P / LA VILLE, DEUXIEME VERSION / $1901: 450$ / ACTE I

c. Je voudrais pouvoir aller à Nantes ou au Havre, comme les débardeurs. PHILIPPE.C-L / LA MERE ET L'ENFANT / $1900: 138$ / CHAPITRE SEPTIèME

d. La répartition des importations par origine révèle en 1937, comme pour les années précédentes, la part prépondérante des pays étrangers INDUSTRIE CONSERVES EN FRANCE /

\subsection{Interprétation}

L'interprétation de la séquence introduite par comme comparatif est toujours propositionnelle, qu'il s'agisse syntaxiquement d'une phrase ou d'une séquence fragmentaire ${ }^{3}$. Au niveau de la construction comparative, la sémantique de comme est constante. Il s'agit d'un opérateur qui prend deux arguments : (1) la sémantique de l'item auquel le syntagme en comme se combine (en (8), l'adjectif souligné) et (2) une proposition, interprétée à partir de la séquence introduite par comme (entre crochets dans les exemples (8)). La relation sémantique dénotée par comme, c'est-à-dire la relation de

\footnotetext{
${ }^{3}$ On distinguera de ce fait les coordinations en comme, dont le conjoint ne s'interprète pas toujours de façon propositionnelle (cf. Mouret \& Desmets, ce numéro) : La France comme l'Angleterre ont accepté de signer le traité.
} 
comparaison, établit un rapport d'identité ou de similitude entre deux propriétés ou deux ensembles de propriétés : propriété(s) de l'argument (1) et propriété(s) de l'argument (2).

(8) a. Marie est belle comme [sa sœur au même âge].

b. Marie est belle comme [sa sœur était (x)belle au même âge].

Dans cette relation, la proposition interprétée à partir de la séquence introduite par comme fournit la propriété ou l'ensemble de propriétés à comparer; on abstrait la propriété en question ${ }^{4}$. Dans les formes pleines, cette propriété correspond à un constituant extrait (un adverbe, représenté par $x$ en (8)). Son interprétation n'est pas explicite, elle est construite à partir du type sémantique de son argument (propriété ou événement) et de la sémantique lexicale de cet argument. Ce qui explique que l'on obtienne de la manière (parler comme un poète), de l'intensité (bleu comme l'azur) ou bien du degré (grand comme Marie). Dans le cas de la comparative d'analogie, ajout à une phrase, on peut penser que la comparaison examine une propriété commune aux deux propositions (tout comme Paul va an foot le mercredi soir, Marie va an tennis tous les samedis) qui correspond à la valeur de vérité, ou à la « satisfiabilité » (cf. Mouret \& Desmets, ce numéro).

\section{Propriétés des phrases elliptiques en comme}

Les phrases elliptiques sont des phrases dont le verbe tête est absent, qui réalisent un certain nombre d'éléments, appelés éléments résiduels, et dont l'interprétation et la forme dépendent d'une phrase complète présente dans le contexte immédiat. L'ellipse est un phénomène d'interface qui met en jeu des contraintes de parallélisme syntaxiques, sémantiques et pragmatiques.

Ce phénomène apparaît dans plusieurs contextes syntaxiques, mais nous portons ici notre attention sur les phrases elliptiques des constructions comparatives et des constructions coordonnées.

\subsection{Propriétés générales des phrases elliptiques ${ }^{5}$}

L'apparition de l'ellipse est conditionnée par un environnement sémantique et pragmatique particulier. Minimalement, deux propositions, $\mathrm{P}$ et $\mathrm{Q}$, sont associées par une certaine relation sémantique (coordination, comparaison), voire par une certaine relation discursive (en particulier pour la coordination ou la juxtaposition : cause-effet,

\footnotetext{
4 On peut expliciter la relation sémantique associée à comme dans la phrase il est grand comme son frère est petit par le schéma suivant (d'après Desmets 2001) : il existe deux états, s1 et s2, ayant chacun une propriété, appelons-là "Qualité », respectivement $\Phi$ et $\Psi$, et $\Phi$ et $\Psi$ sont identiques, ou encore s1 et s2 sont $\Phi$-comparables. On pourra formaliser l'interprétation de la relation de comparaison (de Qualité d'états) de la manière suivante : grand (s1, x) \& petit (s2, y) \& $\Phi(\mathrm{s} 1) \& \Psi(\mathrm{s} 2) \& \lambda \mathrm{s} \Phi(\mathrm{s})=$ $\lambda_{s} \Psi(\mathrm{s})$; l'abstracteur rend compte du fait que $\Phi$ et $\Psi$ sont des prédicats appropriés au type de variable $\mathrm{s}$ (《 $\mathrm{s}$ » étant une variable associée au type « état»).

${ }^{5}$ Pour une présentation générale des phrases elliptiques et fragmentaires, se reporter à Abeillé (à paraitre).
} 
aboutissement narratif, etc., cf. Kehler 2002). P et Q présentent un certain nombre d'éléments similaires, en particulier, le prédicat tête (i.e., la relation sémantique principale) de Q est le même qu'un des prédicats de P. L'ellipse intervient dans la séquence dénotant $Q$ et permet de ne pas réaliser les éléments déjà présents dans $P$. Aussi, peut-on dire que la phrase contenant $\mathrm{P}$ fournit le contexte linguistique permettant l'interprétation des éléments non réalisés dans la séquence elliptique (Q). On appelle la phrase qui contient $\mathrm{P}$, phrase antécédent ou phrase source, et phrase cible, la séquence elliptique $(\mathrm{Q})^{6}$ :

(9) a. P [Jean est brun] et Q [Marie est blonde].

b. $\mathrm{P}$ [Jean aime autant le bricolage] que $\mathrm{Q}$ [Marie aime la décoration].

c. $\mathrm{P}$ [Jean aime le bricolage], comme $\mathrm{Q}$ [Marie aime la décoration].

Du fait que sémantiquement le prédicat reconstruit de la phrase cible est identique à celui de la phrase source, les éléments de la valence syntaxique du verbe absent ne peuvent être que catégoriellement identiques ou très similaires; ce qui contribue à l'effet de parallélisme. On verra plus bas que d'autres contraintes de bonne formation syntaxiques sont à respecter.

Le phénomène de l'ellipse est tenu pour être syntaxiquement optionnel (cf. Chomsky, 1977). Dans cette perspective, seules les conditions pragmatiques et les stratégies discursives liées à la répétition détermineraient la possibilité de son effectuation, et, le cas échéant, le locuteur aurait la possibilité de réaliser des éléments d'information similaires.

(10) a. p[Jean est brun], Q[Marie est blonde].

b. $\mathrm{P}[$ Jean est plus brun] que $\mathrm{Q}$ [Marie n'est blonde].

c. P[Marie est belle] comme $\mathrm{Q}$ [était belle sa sœur au même âge].

Dans le cas général, il est vrai, le locuteur ne réalise en Q que l'information minimale et différente de $\mathrm{P}$ - information pertinente au sens où elle fournit des arguments distingués dans le calcul sémantique de la coordination ou de la comparaison (Amsili \& Desmets, 2008a). Ceci ne va pas sans entraîner de possibles ambiguités sémantiques ${ }^{7}$, en général levées par le contexte pragmatique. Toutefois, la

${ }^{6}$ A titre indicatif, le prédicat que l'on peut interpréter à partir de la phrase antécédent est écrit en italique indicé. Il ne s'agit pas d'une reconstruction syntaxique.

${ }^{7}$ Les ambigüités en comparatives sont celles repérées depuis longtemps pour les coordinations (Hankhamer 1971) : (a) identité souple vs stricte : ou bien Pierre donne des roses à sa femme (identité souple) ou bien Pierre donne des roses à la femme de Jean (identité stricte).

(i) Jean donne plus de roses à sa femme que Pierre.

(ii) Jean donne des roses à sa femme, comme Pierre.

(b) ambiguité de fonction du constituant résiduel : En (iii), ou bien Marie connaît mieux Sarah ou bien Jean connait mieux Marie ; en (iv) avec une prosodie continue sur l'ajout comparatif, on obtient une lecture de type coordination (cf. Mouret \& Desmets, ce numéro) : Jean connait Sarah et Marie, en (v) avec une prosodie détachée on rétablit l'ambigüité sur la fonction du constituant Marie qui peut être sujet ou objet.

(iii) Jean connait Sarah mieux que Marie. 
répétition à l'identique peut être syntaxiquement impossible, lorsqu'elle bloquée, par exemple, par la forme du pronom personnel sujet :

(11) Paul fera la vaisselle lundi, comme moi *ferai la lessive mardi.

De la même façon, la reconstruction syntaxique du verbe peut être interdite pour des raisons lexicales. Par exemple, la conjonction de coordination ainsi que n'admet pas de verbe fini :

(12) Paul viendra, ainsi que Marie *viendra.

Ces deux arguments montrent que l'ellipse n'est pas un phénomène syntaxiquement optionnel.

Inversement, ainsi que l'a repéré Zribi-Hertz (1986), il ne suffit pas que deux propositions soit associées pour permettre une ellipse. Le phénomène est exclu dans d'autres subordonnées ajouts et n'est pas possible dans toutes les coordinations :

(13) Jean boit du thé *quand / *si /*parce que/*car Marie du café.

Par conséquent, la phrase elliptique n'est pas seulement subordonnée à des conditions sémantiques et pragmatiques, elle connait également des contraintes syntaxiques.

\subsubsection{Absence du verbe tête}

Dans les cas que nous étudions, l'ellipse intervient minimalement mais obligatoirement sur le verbe tête de la séquence fragmentaire $(\mathrm{Q})^{8}$ :

(14) *Jean mange des pommes et Marie mange (despommes).

Ce n'est pas la catégorie verbale en soi qui est sensible au phénomène, mais la fonction tête. Ainsi, dans le cas d'un verbe à un temps composé, l'auxiliaire peut seul être omis :

(15) a. P [Jean a bu un fond de champagne] et $\mathrm{Q}$ [mangé le gâteau qui restait].

b. $\mathrm{p}$ [Jean a plus aimé la pièce] que $\mathrm{Q}$ [détesté].

c. $\mathrm{p}$ [Jean a grimpé], comme $\mathrm{Q}$ [descendu les pentes à vive allure].

De même, lorsque le verbe tête de $\mathrm{Q}$ sous-catégorise un complément verbal à l'infinitif, ce dernier peut être réalisé si la tête verbale, elle, ne l'est pas (16a,c,e) ; en revanche, si la tête verbale est réalisée, son verbe infinitif complément, lui, ne peut pas être ellipsé $(16 \mathrm{~b}, \mathrm{~d}, \mathrm{f})$ :

(iv) Jean connait Sarah comme Marie.

(v) Jean connaît Sarah, comme Marie.

(c) ambiguité sur l'antécédent: ou bien l'ellipse correspond à la phrase «Paul dit qu'il va à la montagne » ou bien à la phrase «Paul va à la montagne ».

(vi) Jean dit qu'il va à la montagne plus souvent que Paul.

(vii) Jean dit qu'il va à la montagne comme Paul.

8 Les dépendants de $\mathrm{V}$ ont fréquemment une réalisation non canonique, une omission: (i) Jean mange plus de pommes que Marie ne mange.; (ii) il est arrivé comme c'était prévu. Ou bien une forme de résomption : (i) Idiot comme il l'est. 
(16) a. Marie se débrouille pour aller au marché le mercredi et Pierre pour y aller le samedi.

b. *Marie se débrouille pour aller au marché le mercredi et Pierre se débrouille le samedi.

c. Marie aime plus aller au marché le mercredi que Pierre y aller le samedi.

d. *Marie aime plus aller au marché le mercredi que Pierre aime le samedi.

e. Marie aime aller au marché le mercredi, tout comme Pierre y aller le samedi.

f. *Marie aime aller au marché le mercredi, tout comme Pierre aime le samedi.

Une conséquence de l'absence obligatoire du verbe tête de Q est que l'ellipse ne peut apparaittre dans une phrase enchâssée à l'intérieur de $\mathrm{Q}^{9}$ :

(17) a. *Marie va au marché le mercredi et $\mathrm{Q}$ [ je crois que [Pierre le samedi ]].

b. *Marie va plus souvent au marché le mercredi que $\mathrm{Q}$ [je crois que [Pierre le samedi ]].

c. *Marie va souvent au marché le mercredi, tout comme Q[ je crois que [Pierre le samedi ]].

\subsubsection{Propriétés des constituants résiduels}

Il peut y avoir plus d'un constituant résiduel dans les phrases elliptiques, et les catégories réalisées sont diverses : syntagmes nominaux, prépositionnels ${ }^{10}$, adjectivaux, adverbiaux, et verbes non tensés. Les pronoms personnels faibles sont évidemment exclus ${ }^{11}$. Il s'agit d'éléments clitiques nécessitant la présence contiguë d'un verbe ; à la place figurent des pronoms forts.

(17) a. Nous sommes allés au cinéma, Paul et *je/moi.

b. J'aime plus repasser que $*_{\text {tu/toi. }}$

c. J'adore aller au cinéma, comme $*$ il/lui.

Des liens syntaxiques sont actifs dans la séquence elliptique : la fonction des éléments résiduels s'interprète comme s'il s'agissait d'une séquence verbale finie : en (18a), sujet et complément direct ; en (18b), sujet et complément oblique ; en (18c), complément direct et SP oblique et SP ajout; en (18d), sujet et SN ajout; en (18e), sujet et N' quantifié complément direct; en $(18 \mathrm{f}, \mathrm{g})$, sujet et attribut ; circonstancielles ajouts en $(19)^{12}$.

(18) a. et je l'ai obtenue contre une boule de pain, une bougie, et une pièce de vingt sous neuve dont la clarté a dissipé, comme le soleil un brouillard, les hésitations dernières de la vieille. GENEVOIX.M

\footnotetext{
${ }^{9}$ Il est en revanche possible de trouver une ellipse dans une enchâssée si le $\mathrm{SN}$ résiduel apparait avec un adverbe additif (je crois que Paul aussi), ou polaire (Paul viendra mais je pense que Marie pas/non), qui semblent fonctionner comme une anaphore prédicative semblable aux formes so ou do so de l'anglais (voir Miller 1992 et plus largement l'ensemble de la littérature sur l'ellipse du SV en anglais).

${ }^{10} \mathrm{Il}$ est à noter que le constituant résiduel peut également être une préposition forte seule : Plus de personnes ont voté pour la proposition que contre; Difficile de tenir cette boîte fermée, qu'on la prenne par dessus comme par dessous.

${ }^{11}$ En anglais, les pronoms personnels sujets, qui ne sont pas faibles, ne sont pas exclus. Dans : Who wants to answer? - Not I/me.

${ }^{12}$ En outre, la forme de l'ellipse est insensible au type de fonction qu'occupe l'ajout comparatif en comme ou en que.
} 
b. Cette année, Jean a plus souvent rendu visite à Marie à Paris que Pierre à Bologne.

c. Cette année, les gens pourront voir l'exposition à Rome en juillet comme à Paris en septembre.

d. Celle qui court le long de votre vie comme le feu le long d'un cordon Bickford, qui ne sait allumer que des coups de tête, de pauvres et bruyants pétards? BAZIN.H / LA MORT DU PETIT CHEVAL / $1950: 24$ / II

e. Jean gagne plus de coupes que Pierre de médailles.

f. Jean est plus agréable que Marie serviable.

g. Jean est agréable comme intelligent, et serviable par dessus tout.

(19) a. Il m'a parlé comme si c'était la première fois.

b. Ferme la porte comme quand tu pars en vacances.

c. Pierre était autant embarrassé que si on lui avait découvert une maitresse.

d. Pierre était autant gêné que quand on l'a rencontré chez Claire l'autre jour.

L'ordre linéaire (20a,b), la catégorie (20c,d), le nombre exact des constituants (20e,f) ne sont pas obligatoirement identiques à ceux des constituants de la phrase antécédent. Abeillé \& Godard (1996), puis Mouret (2006) l'ont montré pour la coordination ; il en est de même pour les constructions comparatives :

(20) a. On a plus promu la voiture à Paris qu'à Londres les transports en commun

b. Le dynamisme des entreprises attire les investissements à Londres, comme à Paris l'industrie du luxe.

c. Paul aime plus les longues soirées entre amis que passer des heures à méditer en pleine nature.

d. Paul aime les longues soirées entre amis, comme passer des heures à méditer en pleine nature.

e. Paul a plus souvent écrit des petits mots doux à sa mère que des poèmes de grande valeur.

f. Paul a écrit des petits mots doux à sa mère, comme des poèmes de grande valeur.

En revanche, les constituants réalisés doivent correspondre à une valence possible du verbe antécédent.

(21) a. *Paul pratique autant le golf que Marie faire les magasins. (Mouret)

b. *Paul pratique le golf comme Marie faire les magasins.

Ainsi, les séquences elliptiques de (21) sont rejetées parce que le V antécédent pratiquer ne sous-catégorise pas de SV infinitif complément ; les phrases obtenues en réalisant le matériel manquant seraient agrammaticales.

\subsection{Construction comparative vs construction coordonnée}

Il existe un certain nombre de points communs entre les constructions comparatives et les constructions coordonnées. Les contraintes de parallélisme sémantique et syntaxique sont les plus notables, elles expliquent que l'ellipse soit présente dans les deux cas. Toutefois, il s'agit de deux constructions syntaxiquement bien distinctes, comme le montre l'examen des propriétés des phrases elliptiques. 


\subsubsection{Parallélisme syntaxique et sémantique}

Dans le cas de la coordination de propositions, le parallélisme constructionnel provient d'une part de la sororité syntagmatique des constituants (i. e., ils sont structurellement au même niveau), d'autre part de la sémantique: lorsque des propositions sont coordonnées on obtient l'abstraction d'une archi-propriété à partir d'un ensemble d'alternatives ne se distinguant potentiellement que par une différence minimale.

Dans le cas de la comparaison, la réitération d'éléments de la phrase source, laquelle contribue à l'effet de parallélisme, est liée à la sémantique de la construction (cf. Amsili et al., à paraittre). La comparaison discrimine deux éléments, qu'elle examine sous l'angle d'une de leurs qualités ou propriétés respectives. Ce qui donne lieu à une relation d'ordre (inférieur/supérieur/égalité pour la comparaison scalaire) ou à une relation d'égalité ou de similarité (pour la comparaison de qualité en comme). Pour que l'opération soit menée à bien, il faut satisfaire certaines conditions, entre autres, s'assurer que les deux éléments sont propres à la comparaison : ils doivent présenter un minimum de traits communs (mais peuvent en présenter un maximum; logiquement, une différence suffit), en particulier, ils doivent avoir le même type sémantique (deux propriétés, deux événements, deux états, deux entités, etc.). Excepté si la comparaison porte sur le procès lui-même (ce qui n'engendre pas d'ellipse), la relation prédicative principale de la comparative est identique à celle de la phrase source (qui construit la description de l'élément comparé).

Les comparatives en comme possèdent en propre des contraintes de parallélisme qui proviennent directement de leur structure syntaxique ${ }^{13}$. Ces dernières s'analysent, en effet, comme des relatives sans antécédent (Desmets 2001, ou « intégratives » pour Le Goffic 1991). Il s'agit d'une structure à dépendance non bornée qui enregistre l'extraction d'un adverbe (de type manière), arrêtée par comme, adverbe qu-. Comme assume deux rôles, celui de borne pour la dépendance et celui de tête d'un syntagme adverbial. Le parallélisme provient d'une tendance bien connue des relatives sans antécédent, qu'on nomme « coïncidence fonctionnelle 》 (Bresnan \& Grimshaw 1978), et qui s'exprime ici par le fait que l'adverbe manquant (extrait) a en général une fonction similaire à (ou en correspondance avec) la fonction de la relative dans son ensemble. La répétition du même lexème assure cette similarité fonctionnelle.

(22) a. Chez Marie, Paul se comporte [comme un éléphant (se comporterait_) dans une boutique de porcelaine].

b. ?Chez Marie, Paul se comporte [comme un éléphant marcherait_dans une boutique de porcelaine].

c. Dans cette situation, Jean est gêné [comme parfois les gens sont ?embarrassés/gênés_devant trop de générosité].

\footnotetext{
${ }^{13}$ Il existe également un effet de parallélisme syntaxique dans les comparatives scalaires, dans la mesure où l'adverbe de degré extrait de la phrase en que a une fonction similaire à celle de l'adverbe de degré réalisé dans la phrase matrice (cf. Amsili \& Desmets, 2008a) : Pierre a plus aimé les acteurs que Sarah n’a $(x)$ aimé la mise en scène.
} 
d. */??Dans cette situation, Jean est gêné [comme parfois les gens se comportent_ devant trop de générosité].

En (22), les phrases dans lesquelles la coïncidence fonctionnelle n'est pas respectée (22b,d) ont une acceptabilité dégradée par rapport à celles où la coïncidence est là : comparative complément de $\mathrm{V}$ avec extraction d'un adverbe complément de $\mathrm{V}$ en (22a) et comparative ajout modifieur d'A avec extraction d'un adverbe modifieur d'A en (22c).

\subsubsection{Les comparatives ne sont pas des coordinations}

Bien qu'elles offrent toutes deux la possibilité d'une séquence elliptique, les constructions comparatives ne sont pas des constructions coordonnées, contrairement à ce qui est parfois admis ${ }^{14}$. Elles montrent des différences syntaxiques importantes, concernant l'ordre des mots et les conditions sur l'extraction, qui modifie la distribution de leurs phrases elliptiques.

\section{Place de la séquence elliptique}

En coordination, la séquence fragmentaire ne peut précéder la phrase source ; cette contrainte ne s'applique pas dans le cas d'une construction comparative ${ }^{15}$ :

(23) a. *Jean une pomme et Marie mange une poire

b. Comme Paul les jours de pluie, Marie se décide à mettre des bottes aujourd'hui.

c. Le monde social est parsemé de rappels à l'ordre qui ne fonctionnent comme tels que pour les individus prédisposés à les apercevoir, et qui, comme le feu rouge le freinage, déclenchent des dispositions corporelles. Bourdieu (Méditations pascaliennes: 210)

La place de la séquence elliptique dépend du type de construction dans lequel elle apparait. Dans une coordination, la phrase source est obligatoirement dans le premier conjoint. En revanche, la relation syntaxique entre l'ajout comparatif adverbe de phrase et la phrase hôte autorise une interprétation 'à rebours'. Selon Marandin (1999), les ajouts sont, en effet, mobiles dans le domaine syntagmatique auquel ils s'adjoignent, ce qu'on vérifie dans le cas des comparatives, mais ils ne peuvent être réalisés en dehors. Les comparatives en comme adverbes de phrase peuvent être antéposées à la phrase hôte (comme en $23 \mathrm{~b}$, ou en $24 \mathrm{a}$ ), un ajout modifieur de SV peut être

\footnotetext{
14 On n'inclura donc pas ici la coordination en comme présentée dans Mouret \& Desmets (ce numéro).

15 On signale que les comparatives scalaires modifieurs présentent leurs propres contraintes de placement (cf. Amsili \& Desmets, 2008b). En revanche, les scalaires parenthétiques ajouts à la phrase matrice peuvent être antéposées :

(i)a. Plus que Betty, Anne a eu peur de l'accident

b. ?Plus que Betty la vitesse, c'est le choc qui a bouleversé/surpris Amy.

c. Plus que des subventions aux cités, il faut donner du travail aux jeunes.

d. Il faut, plus que des subventions aux cités, donner du travail aux jeunes.

e. Certains invités ont, moins que d'autres, apprécié le repas de ce soir.
} 
mobile à l'intérieur du SV (24b), mais un ajout modifieur d'A ne peut être réalisé en dehors du SA, comme en $(24 \mathrm{c})$ :

(24) a. Comme son frère, Paul est grand.

b. et je l'ai obtenue contre une boule de pain, une bougie, et une pièce de vingt sous neuve dont la clarté a dissipé, comme le soleil un brouillard, les hésitations dernières de la vieille. GENEVOIX.M / CEUX DE 14 / 1950 : 211 / LIVRE II NUITS DE GUERRE 1917, II NOTRE PATELIN : MONT-SOUS-LES-CôTES

c. (Paul est grand comme son frère.) *Comme son frère Paul est grand.

\section{Contraintes sur l'extraction}

Parmi les contraintes de parallélisme syntaxique repérées en structures coordonnées figure la généralisation de Wasow (Ross 1967, Pullum et Zwicky 1986) qui impose, en particulier, à chaque conjoint de respecter des contraintes d'extraction (i.e., s'il y a extraction dans l'un des conjoints, il y a obligatoirement extraction dans l'autre; la contrainte est dite across the board) :

(25) a. En ce moment, la Bible et le roman de cet auteur se vendent bien.

b. *Voilà un auteur dont la Bible et le roman_ se vendent bien.

c. Voilà un auteur dont l'essai_ et le roman_ se vendent bien.

Les comparatives n'observent pas la même contrainte. Il est possible d'extraire un constituant de la phrase matrice sans extraire obligatoirement un constituant correspondant de la phrase comparative ${ }^{16}$ :

(26) a. Voilà un auteur dont les romans_se vendent mieux que la Bible.

b. Voilà un auteur dont les romans_se vendent comme la Bible.

c. Voilà un auteur dont les romans_se vendent bien, mieux que la Bible.

d. Voilà un auteur dont les romans_ se vendent bien, comme la Bible.

Les comparatives montrent une dissymétrie que n’autorisent pas les coordinations.

On observe, en revanche, un autre phénomène. On ne peut extraire de constituant de la séquence introduite par que comparatif ou par comme sans qu'un constituant correspondant dans la phrase matrice ne soit lui-même extrait. Les syntagmes comparatifs ajouts à V/SV en (27-28) et ajouts à $\mathrm{P}$ (parenthétiques) en (2930) que nous testons ici présentent le même comportement :

(27) a. Voilà un auteur dont le dernier roman_ s'est vendu comme les nouvelles_ qu'il a publiées l'année passée.

b. *Voilà un auteur dont la Bible se vend comme les romans_.

(28) a. Voilà un auteur dont le dernier roman_s'est mieux vendu que les nouvelles_qu'il a publiées l'année passée.

b. *Voilà un auteur dont la Bible se vend mieux que les romans_.

(29) a. Voilà un auteur dont le dernier roman_ s'est bien vendu, comme les nouvelles_qu'il a publiées l'année passée.

d. *Voilà un auteur dont la Bible se vend bien, comme les romans_.

16 Ainsi que l'a noté Williams (1977) pour l'anglais, les comparatives ne seraient pas un contexte de type across the board pour l'extraction. 
(30) a. Voilà un auteur dont le dernier roman_ s'est bien vendu, mieux que les nouvelles_ qu'il a publiées l'année passée.

b. *Voilà un auteur dont la Bible se vend bien, mieux que les romans_.

En français, les comparatives (en que ou en comme) sont des îlots pour l'extraction (i.e., a priori, on ne peut pas extraire de constituant en dehors de ces constructions, cf. Desmets, à paraittre). Mais, cette contrainte est levée dans les cas limités où un constituant extrait est en relation avec un constituant manquant de la phrase matrice, c'est-à-dire, dans les cas d'une extraction parasite (parasitic gap). Contrairement à l'anglais, l'extraction parasite est assez restreinte en français. Elle donne lieu à des jugements variables selon les locuteurs avec des infinitives (cf. 31a,b); mais elle est habituellement interdite (pour tous les locuteurs) lorsque l'ajout contient une phrase finie (cf. 31c,d) :
(31) a. \%C’est un livre qu'il a soigneusement rangé_ après avoir lu_ .
b. \%Voici un homme dont la femme_ est partie sans prévenir_.
c. *C'est un livre qu'il a soigneusement rangé_ après qu'il a lu -.
d. *Voici un homme dont la femme_ est partie sans qu'elle prévienne_.

L'extraction parasite dans les comparatives fragmentaires est un argument en faveur de l'hypothèse qu'une phrase elliptique ne contient pas de verbe (vide) dans sa structure syntaxique, et qu'il ne s'agit donc pas d'une phrase finie - ce que nous soutenons. Elle donne de mauvais résultats lorsque la comparative ajout présente une phrase finie ${ }^{17}$ :

(32) a. ??Voilà un auteur dont le dernier roman_s'est vendu comme les nouvelles_ qu'il a publiées l'année passée s'étaient vendu, c'est-à-dire absolument pas.

b. Voici un vieillard dont le teint_ est blanc comme (*sont) les cheveux_.

c. Un couteau dont la lame_est coupante comme (*est) la pointe_.

\subsection{Les types de phrases elliptiques}

On a distingué traditionnellement, à l'origine dans le cadre des structures coordonnées, plusieurs formes de phrases elliptiques ${ }^{18}$, selon le nombre et le type d'éléments résiduels réalisés. Généralement, on retrouve les même formes dans plusieurs

${ }^{17}$ Par ailleurs, les comparatives en comme compléments de $\mathrm{V}$ ne présentent pas de contrainte sur la double extraction (cf.i,ii), ce qui confirme bien que le phénomène observé est une contrainte sur l'extraction parasite réservée aux ajouts: (i) Un immeuble dont la voisine de Paul se comporte comme la gardienne_lorsqu'un livreur se présente.

(ii) Un secret dont la cousine de Paul se comporte comme une dépositaire illégitime_sur le point d'être dénoncée.

${ }^{18}$ Nous laissons pour une étude ultérieure l'analyse des mises en facteur (Righ node raising (RNR), cf. Hudson 1976), présentes dans les constructions coordonnées et les comparatives, qui réalisent un verbe fini mais ellipsent un dépendant (complément ou ajout) du verbe de la première phrase. Il n'est pas sûr qu'elles soient disponibles pour la comparaison (de manière) en comme, et elles ne semblent possibles qu'avec les comparatives scalaires parenthétiques.

(i) a. Jean apprécie plus qu'il n'aime le théâtre.

b. Jean aime, autant que Marie déteste, les haricots.

c. Jean aime, comme Marie déteste, ces magazines. (coordination en comme vs *manière)

d. Certains souhaitent, comme d'autres redoutent, la mise en place de ces réformes. 
contextes syntaxiques (coordinations, comparaisons, fragments dialogiques - par exemple, les réponses courtes). On distingue, en particulier, les constructions « trouées » (gapping) qui présentent au moins deux constituants dont l'un est interprété comme sujet (Paul est allé an marché, Marie an cinéma); et les fragments unaires (stripping) qui réalisent un constituant, interprété comme un valent (sujet ou complément) ou comme un ajout du verbe, parfois accompagné d'un adverbe (Paul est venu bier, mais pas Marie).

L'examen détaillé montre que ces deux types de phrases elliptiques n'ont pas tout à fait les mêmes propriétés en constructions comparatives et en coordonnées, ce qui confirme à nouveau la différence entre les deux types de constructions. Nous commençons par montrer que les séquences fragmentaires dont les constituants résiduels s'interprètent comme des dépendants du verbe (compléments ou ajouts) ne peuvent être ramenées à une réduction de structures conjointes, que l'on trouve en coordonnées. Puis nous abordons le cas des phrases trouées, et celui des fragments unaires.

\subsubsection{Réduction de structures conjointes (ACC)}

La réduction de structures conjointes, encore appelée coordination de séquences (ou Argument Cluster Coordination, dorénavant ACC), est une séquence dans laquelle le verbe est absent et où les constituants résiduels s'interprètent comme des dépendants, le plus souvent compléments ou ajouts (ou un mélange des deux), du V/SV du premier conjoint. Pour Mouret (2006), à la suite de Zribi-Hertz (1986) et de Gardent (1991), il s'agit d'une coordination de constituants sœurs partageant le même verbe. L'analyse conclut donc qu'il n'y a pas d'ellipse phrastique dans cette séquence de constituants.

Les constituants résiduels d'une ACC correspondent obligatoirement à des constituants sœurs du verbe antécédent (i.e., de même niveau syntagmatique), mais ne sont pas obligatoirement des constituants dits « majeurs » de la phrase source, c'est-àdire immédiatement dominés par le nœud phrase, par le SV racine ou par un SV luimême immédiatement dominé par le SV racine (contrainte de constituance repérée par Hankhamer 1971). En (33a) les constituants résiduels sont majeurs, non en (33b) :

(33) a. Jean dit de rester chez elle à Marie et ??(de rester) ici à Paul. (Abeillé \& Godard 2002)

b. Paul a donné les jouets de sa fille à Marie et *(les jouets) de son fils à Jean. (Mouret 2006)

Dans les constructions comparatives fragmentaires qui présentent des constituants résiduels s'interprétant comme des dépendants du verbe absent, la sororité des syntagmes n'est pas contrainte ${ }^{19}$ :

(34) a. J'ai plus souvent essayé de réviser l'Histoire avec Paul que la Géo avec sa sœur.

\footnotetext{
${ }^{19}$ Les mêmes exemples en coordination (i,ii) ne semblent pas franchement exclus, alors qu'ils devraient l'être selon une analyse ACC. On peut penser que les séquences réalisées ici ne répondent pas à une analyse ACC mais à un cas de gapping, ce qui doit de toutes façons être envisagé pour les cas où l'analyse ACC est exclue parce que les constituants ne peuvent être analysés comme étant sœurs, par ex. en (iii) où le SP extrait du V du premier conjoint rend caduque l'analyse ACC :

(i) ??J'ai proposé à Marie de lui apprendre le grec et à son frère le latin.

(ii) ??Jean dit de réviser son Histoire à Marie et sa Géo à son frère.

(iii) A Marie, Paul donnera un livre demain et un disque à Paul aujourd'hui.
} 
b. J'ai souvent essayé de réviser l'Histoire avec Paul, comme la Géo avec sa sœur.

En interprétant la séquence elliptique en (34) avec le prédicat révisé (que je n'ai révisé la Géo avec sa scur), les constituants la Géo et avec sa scur sont moins enchâssés que ne le sont leurs correspondants dans la phrase matrice avec le prédicat essayé de réviser; les constituants parallèles ne sont donc pas sœurs, ils ne 'partagent' pas le même prédicat. Les exemples (34) montrent qu'il n'y a pas de structure réduite en comparatives.

\subsubsection{Phrases tronées (gapping)}

Les phrases trouées sont une forme de phrase elliptique, que l'on nomme traditionnellement gapping, qui réalise au moins deux éléments résiduels, dont l'un s’interprète comme le sujet du verbe syntaxiquement absent.

\section{Contrainte sur les constituants résiduels majeurs}

Hankhamer (1971), puis Gardent (1991), montrent que les éléments résiduels d'une phrase trouée en structure coordonnée doivent correspondre à des constituants majeurs de la phrase source, comme en (cf. 35a). En (35b), en revanche, le constituant de Pierre n'est pas un constituant majeur au sens défini plus haut.

(35) a. Paul a promis d'essayer d'apprendre le latin et Marie le grec.

b. Paul admire le courage de Marie, et Jean *(le courage) de Pierre. (Mouret 2006)

Les constituants résiduels de la phrase trouée, s'ils doivent être majeurs, ne sont en revanche pas obligatoirement sœurs, ainsi en (35a), on peut interpréter l'ellipse verbale comme étant a promis d'apprendre, les constituants le latin et le grec ne sont alors pas sœurs puisqu'ils sont régis par des prédicats distincts (Marie est moins enchâssé que le grec).

En comparatives, l'ellipse des phrases trouées présente la même contrainte que celle repérée en coordinations : lorsqu'un sujet est réalisé, les éléments résiduels sont obligatoirement majeurs, comme l'illustrent les mauvais résultats de (36) :

(36) a. *Paul a plus souvent donné les jouets de sa fille à Marie que Pierre de son fils à Jean.

b. *Le président a rejeté des propositions du ministre de la justice, comme le viceprésident du ministre de l'économie.

De façon intéressante, cette contrainte est également active lorsque seuls des dépendants de $\mathrm{V}$ (compléments ou ajouts) sont réalisés (cf. 37) ${ }^{20}$ :

(37) a. *Paul a plus souvent donné les jouets de sa fille à Marie que de son fils à Jean.

b. ??Il y a eu plus de propositions du ministre de la justice que de l'économie qui ont été rejetées.

c. *Paul a souvent donné les jouets de sa fille à Marie, comme de son fils à Jean.

b. ??Il y a eu des propositions du ministre de la justice, comme de l'économie qui ont été rejetées.

\footnotetext{
20 On ne peut tester de séquences de constituants sœurs compléments ou ajouts de $\mathrm{V}$ qui ne soient pas majeurs selon la définition énoncée plus haut : ils sont obligatoirement directement dominés par le SV racine ou un V enchâssé sous le SV racine.
} 
Là aussi, les constituants résiduels ne correspondent pas obligatoirement aux constituants sœurs d'un $\mathrm{V}$ de la phrase source ; ce qui est attendu dans le cas où un sujet est réalisé, comme en $(38)^{21}$,

(38) a. Jean dit qu'il voit plus souvent sa mère que Paul la sienne.

b. Jean dit qu'il voit souvent sa mère, comme Paul la sienne.

\section{Forme des constituants antécédents}

Ainsi qu'il a été signalé par Tassin (1998), le gapping en construction coordonnée n'autorise pas l'antécédent d'un constituant résiduel à être un pronom clitique (39a). Une telle contrainte ne s'observe pas en constructions comparatives (39c-e) :

(39) a. Il y a des pommes sur la table. *Paul les mange et Marie les poires.

b. Le paysage le pénétrait comme le soleil cette eau. (Grevisse 12 ème éd. : 170)

c. Gaston, je lui ai parlé aussi souvent que Marie à Paul.

d. Je lui ai plus souvent parlé que Marie à Paul.

e. Paul a acheté des grany, il les aime plus que Marie les golden.

\section{Portée de la négation}

Une différence supplémentaire entre comparatives et coordonnées concerne la reprise ou non de la négation. Alors que la négation n'est pas interprétée dans la phrase trouée des comparatives (40a), elle demande à être reprise dans le second conjoint de la coordination, ce qui, par ailleurs, demande la réalisation de la conjonction $n i$ :

(40) a. Marie ne va pas à la piscine comme Paul à la pêche. (tous les jours, facilement, etc.)

b. ?Marie ne va pas à la piscine et Paul à la pêche.

\subsubsection{Fragments unaires (stripping)}

Les fragments unaires forment un type d'ellipse, appelé stripping ou constructions différées, ne réalisant qu'un constituant résiduel correspondant à un constituant de la phrase source. Il peut être interprété comme un valent du verbe (sujet ou complément) ou bien comme un ajout. En structure coordonnée, le résiduel a la particularité d'être accompagné le plus souvent d'un adverbe (par exemple, pas, même, ou bien, aussi). Le conjoint fragmentaire est mobile dans la phrase source, c'est pourquoi il est analysé comme un ajout incident, plutôt que comme une coordination (Abeillé 2005).

(41) a. Paul viendra, ou (bien) Marie.

b. Paul aime beaucoup, mais pas Marie, ce genre de roman à l'eau de rose.

Dans le cas des constructions comparatives, il n'y a pas de réalisation nécessaire d'un adverbe en plus du constituant résiduel. Le fragment unaire n'est pas limité aux

${ }^{21}$ En (38) si l'ellipse du V s'interprète comme voit, alors les constituants sa mère et la sienne ne sont pas sœurs. 
comparatives ajouts, il peut apparaître avec des comparatives compléments ou attributs :

(42) a. Paul ne se comporte plus comme un enfant de trois ans.

b. Marie est comme toi.

En conclusion, nous avons observé que les comparatives en comme présentent des phrases elliptiques à trouées ou bien unaires. Bien qu'elles reposent sur des conditions de parallélisme relativement similaires, la distribution des ellipses comparatives leur est propre et ne peut être assimilée à celle des constructions coordonnées. Les propriétés structurelles (ordre des phrases, conditions sur l'extraction) des deux types de constructions en témoignent.

\section{Analyse et traitements de l'ellipse}

Dans cette section, nous considérons les enjeux théoriques et les difficultés empiriques à rendre compte de l'ellipse. Nous abordons les difficultés de l'appariement entre phrase source et phrase cible (\$3.1), puis nous évoquons les problèmes qu'une approche par reconstruction syntaxique rencontre à traiter les disparités entre source et cible (\$3.2.). Enfin (\$3.3), nous exposons les avantages d'un modèle non dérivationnel et l'intérêt d'une approche par fragments.

\subsection{Un problème empirique complexe}

L'ellipse est un phénomène complexe qui crucialement met en jeu plusieurs niveaux de représentation linguistiques. Pour les besoins de l'exposé, nous rappelons les formes les plus fréquentes : omission du verbe (43a), du $\mathrm{V}$ et de ses dépendants (43b), du sujet et du verbe (43c).

(43) a. La clarté dissipe les hésitations dernières de la vieille, comme le soleil dissipe un brouillard.

b. Marie se décide à mettre des bottes aujourd'hui, comme Paul se décide à mettre des bottes aujourd'buil

c. Paul aime les longues soirées entre amis, comme Paul aime les heures à méditer en pleine nature, d'ailleurs.

Une grande part des difficultés de traitement de l'ellipse est constituée par l'identification puis l'appariement entre le matériel linguistique de la phrase source utilisé pour interpréter la phrase cible et le matériel fourni par la séquence elliptique (i.e., les constituants résiduels). Syntaxiquement, il faut pouvoir rendre compte de la combinatoire des éléments résiduels (leur linéarisation), leur attribuer une identité fonctionnelle, vérifier qu'ils correspondent (catégoriellement) à une réalisation possible de la valence du verbe omis et s'assurer que les constituants omis et les résiduels sont compatibles. Sémantiquement, il faut construire une proposition en utilisant une relation sémantique verbale associée à un des lexèmes $\mathrm{V}$ fournis par la phrase matrice qui soit compatible avec la sémantique des constituants résiduels. Il faut également avoir accès aux arguments du verbe antécédent qui seront utilisés dans les cas où (cf. b,c) sujet ou dépendants sont absents. Pragmatiquement, pour respecter le sens global associé à la construction elle-même (coordination, comparaison, etc.), il faut 
identifier les informations pertinentes données par la phrase antécédent (i.e., les informations anciennes) et les associer aux informations nouvelles données par les résiduels.

Une autre source de difficultés pour modéliser le phénomène provient de l'absence du verbe tête. Les langues dans lesquelles l'ellipse a été le plus étudiée sont largement configurationnelles (au sens de Bresnan 2000) et endocentriques. Or, le phénomène viole les règles d'organisation canoniques, puisque certaines informations liées à la constituance sont conservées (par exemple, la fonction des résiduels, ou le fait que seuls des constituants majeurs sont résiduels) alors que le recteur principal (la tête verbale) est obligatoirement absent.

Enfin, l'appariement est rendu plus délicat encore lorsque le parallélisme structurel (ou linéaire), catégoriel et/ou morphosyntaxique entre les termes des phrases source et cible n'est pas strictement observé.

\subsection{Dissymétries entre phrase source et séquence cible}

\subsubsection{Problèmes de l'approche par reconstruction}

Face aux ellipses de type gapping l'approche de la Grammaire Générative et transformationnelle depuis (Hudson 1976, Ross 1967) jusqu'à Kennedy \& Merchant (2000) et Merchant (2004) est de postuler l'existence en structure profonde d'une structure phrastique dont certains constituants n'ont pas de réalisation en surface ; la structure de la phrase elliptique est obtenue par dérivations successives à partir d'une copie de la structure de la phrase source et effacement des constituants non phonétiquement réalisés dans la phrase cible.

Bien que l'idée qu'elle met en œuvre soit assez conforme à l'intuition, ce type d'approche pose des problèmes théoriques et empiriques. Tout d'abord, il est gênant de postuler une structure syntagmatique verbale vide. D'une part, la phrase elliptique se comporte comme une catégorie non finie (cf. l'omission obligatoire du verbe tête, et le résultat de l'extraction parasite). D'autre part, du point de vue théorique, on justifie mal que deux types de phénomènes bien distincts que sont les ellipses (qui ne correspondent pas à un vide sous-catégorisé) et les vides syntaxiquement obligatoires ('extraction d'un constituant, par exemple) reçoivent la même représentation.

En outre, la copie de la phrase source est problématique. Historiquement, les études portant sur l'ellipse ont majoritairement concerné les structures coordonnées, et, étant donné les contraintes de parallélisme de ces constructions, plus fortes qu'en comparatives, on comprend le recours à la copie. Cependant, l'opération suppose l'intégrité formelle des constituants et de la structure (i.e., de la configuration syntagmatique) à recopier, alors que les données que nous observons ne montrent pas forcément d'identité formelle avec le matériel de la phrase source. Le seul recours dans ce type de cadre théorique pour traiter la disparité des réalisations de surface est une multiplication des cycles de dérivations sémantiques, morphologiques et syntaxiques, ce qui, en plus d'être coûteux, entraine potentiellement des incohérences théoriques. 


\subsubsection{Difficultés empiriques}

La gestion de l'appariement entre phrase source et phrase cible est un enjeu pour tous les modèles. Dans cette section nous rassemblons les aspects empiriques les plus problématiques. Les données qui suivent montrent en particulier qu'on ne peut inscrire structurellement cette répétition, ni envisager une duplication des formes instanciées dans la phrase antécédent car les propriétés morphosyntaxiques ne sont pas toujours conservées.

\section{Propriétés structurelles}

Ainsi que nous avons pu l'observer, il arrive que les constituants résiduels et leurs correspondants dans la phrase source n'aient pas de propriétés syntaxiques identiques. Par conséquent, on ne peut pas s'appuyer sur la structure syntaxique de la première phrase pour générer celle de la séquence elliptique (cf. Culicover \& Jackendoff 2005, pour l'anglais). Ci-après, nous rappelons les faits.

Tout d'abord, la catégorie des constituants mis en parallèle peut ne pas être la même ; comme ici un SN et un SV compléments (44).

(44) Paul aime les longues soirées entre amis comme passer des heures à méditer en pleine nature.

Les propriétés structurelles des constituants peuvent être distinctes, comme ici, lorsque le verbe antécédent enregistre l'extraction d'un complément du N du SN objet sans qu'il y ait d'extraction correspondante dans la phrase cible (si l'on devait la reconstruire syntaxiquement) :

(45) a. Un auteur dont on vend mieux les romans _que la Bible. (\#qu'on ne vend la Bible de cet auteur)

b. Un auteur dont les romans_se vendent comme la Bible. (\#de cet auteur se vend)

L'ordre des constituants résiduels peut être différent de celui des constituants correspondants de la phrase source; SN objet - SP locatif /SP locatif - SN objet en (46a); SN sujet - SP locatif / SP locatif - SN sujet (46b) :

(46) a. On a plus promu la voiture à Paris qu'à Londres les transports en commun

b. Le dynamisme des entreprises attire les investissements à Londres, comme à Paris l'industrie du luxe.

Le nombre des constituants peut être différent, les valences peuvent ne pas être identiques ; SN objet - SP à complément/ SN objet (47) :

(47) a. Paul a écrit des petits mots doux à sa mère, comme des poèmes de grande valeur.

b. Paul a plus souvent écrit des petits mots doux à sa mère que des poèmes de grande valeur.

Les constituants mis en correspondance peuvent être sous-catégorisés par des prédicats structurellement différents : en (48), le prédicat antécédent est a promis d'essayer 
d'apprendre le latin alors qu'on pourrait reconstruire la phrase comparative avec le prédicat a promis d'apprendre le grec.

(48) a. Paul a plus souvent promis d'essayer d'apprendre le latin que Marie le grec.

b. Paul a souvent promis d'essayer d'apprendre le latin, comme Marie le grec.

Enfin, la mobilité de la comparative d'analogie dans la matrice pose une difficulté supplémentaire aux modèles qui ne dissocient pas constituance et linéarisation des constituants. Non seulement il faut pouvoir traiter les cas d'antéposition, comme en (49a), mais encore, il faut autoriser les cas de 'scramble', c'est-à-dire d'insertion incidente, comme en (49b):

(49) a. Tout comme son frère, Paul est grand.

b. Paul, comme son frère, va être très grand.

\section{Propriétés morphosyntaxiques}

Il n'y a pas toujours identité de forme entre le verbe antécédent et celui qui serait réalisé, dans l'hypothèse d'un effacement ultérieur, dans la séquence elliptique. Un modèle dérivationnel se trouve face à un problème d'indécision, qui ne peut être résolu pour chaque cas de figure que nous citons, que par une multiplication de règles particulières pour obtenir la forme verbale cible attendue.

Ainsi, dans les constructions comparatives suivantes, le mode des verbes peut être différent. En $(50 \mathrm{a}, \mathrm{b})$ la présence de l'ajout hypothétique impose un verbe tête au conditionnel. En (50c,d), la séquence manquante est soit au présent générique soit au conditionnel ${ }^{22}$, et peut même présenter une modalité (powvoir, avoir l'babitude, etc.) qui n'était pas présente en phrase source :

(50) a. Il m'a parlé comme il m'aurait parlé si c'était la première fois.

b. Pierre était aussi embarrassé qu'il le serait si on lui avait découvert une maitresse.

c. Ah non, tu ne vas pas te plaindre de tout comme Pierre! comme Pierre se plaint/ a l'habitude de se plaindre / se plaindrait s'il était là, etc.

d. Manger comme un cochon, ça ne lui ressemble vraiment pas. comme un cochon mange / mangerait / pourrait manger, etc.

D’une part, il n'y a pas duplication du verbe tel qu'il est instancié dans la phrase pleine, d'autre part, l'idée même d'une reconstruction syntaxique se heurte à l'aléatoire de la forme que peut prendre le verbe; il n'est pas possible de prédire quelles/toutes les modalités réalisables dans cet environnement.

Le temps grammatical du verbe, également, peut varier ${ }^{23}$, modulo certaines contraintes pragmatiques qui doivent être satisfaites pour motiver l'association entre les situations décrites dans la phrase source et la phrase cible. En (51a-c), le temps

22 Ce sont des modes caractéristiques de la construction à parangon (Milner 1973). En outre, si un opérateur de négation est présent sur le verbe de la principale, il peut ne pas être repris ou interprété dans la subordonnée ajout.

${ }^{23}$ La coordination montre des contraintes d'identité plus stricte en ce domaine (cf. Mouret 2007). 
grammatical est imposé par la présence d'un adverbe temporel; en (51d,e), il est pragmatiquement justifié parce que le comparant véhicule une vérité établie ou une connaissance générale ${ }^{24}$ :

(51) a. (Amy n’a pas assez répété son examen) - Rassure-toi, Amy travaillera plus longtemps chez elle demain que chez toi hier.

b. Paul va de mieux en mieux, demain, il mangera comme avant.

c. L'aptitude de l'ancêtre à grimper dans les arbres devait le mettre à l'abri, comme aujourd'hui les singes, des grands prédateurs. LE MONDE : 08/02/01: 25

d. La salle entière chavira sous ses yeux, glissant avec lenteur de droite à gauche comme le pont d'un navire sur une mer démontée. GREEN.J / MOIRA / 1950 : 42 / PREMIèRE PARTIE

e. Tu seras laide comme les quatorze péchés capitaux !

En structures coordonnées, comme en comparatives, on assiste à une neutralisation des phénomènes d'accord ; le verbe de la séquence source et celui que l'on restituerait dans la séquence elliptique ne portent pas obligatoirement le même nombre :

(52) a. Demain au restaurant, Paul mangera des lasagnes et les enfants des panini.

b. Demain au restaurant, Paul mangera autant de lasagnes que les enfants de panini.

c. Demain au restaurant, Paul mangera des lasagnes, comme les enfants des panini.

Quel que soit le modèle, il faut pouvoir associer (ou générer) des verbes dont la réalisation morphosyntaxique en termes de valence présente des différences. En imaginant que l'on reconstruise le verbe manquant, on observe en (53) une forme verbale 'prodrop' (impératif) ou une forme non finie (infinitif) dans la phrase matrice et une forme avec un pronom personnel sujet (clitique) dans la comparative :

(53) a. Ferme la porte comme * (tu) fermes la porte quand tu pars en vacances.

b. J'ai essayé de danser comme toi tu danses, ça ne marche pas.

En (53b) la forme du constituant résiduel, un pronom personnel fort, empêche tout simplement la reconstruction littérale d'une phrase finie. La seule façon d'en reconstruire une syntaxiquement est de postuler une dislocation du pronom fort et une forme verbale avec clitique correspondant. Le cas est identique en (54) :

(54) a. Paul aime faire la vaisselle comme *moi aime faire la lessive

b. Paul aime faire la vaisselle comme moi j'aime faire la lessive

A l'inverse, ainsi que signalé plus haut, une forme verbale cliticisée (55a), ou bien encore, une forme verbale ne réalisant pas un constituant (i.e., une forme 'gapée') (55b) dans la phrase matrice peut être mise en parallèle avec une forme verbale réalisant syntagmatiquement le constituant correspondant :

(55) Le paysage le pénétrait comme le soleil cette eau.

A leurs yeux, l'absolue domination sur le golf mondial de celui qui multiplie les victoires comme d'autres les pains, parfois miraculeusement il faut le reconnaître, est une preuve irréfutable de sa nature divine. (Le Monde portail web du 11/04/2008)

\footnotetext{
${ }^{24}$ Sur la valeur générique des ajouts d'analogie, voir Moline (à paraître).
} 
En guise de conclusion, on ajoutera les deux exemples suivants plusieurs fois problématiques. En (56), il faut résoudre le problème posé par l'insertion incidente (les informations à utiliser se trouvant de part et d'autre), et, sachant qu'on peut avoir des inversions de sujet $\mathrm{SN}$, s'assurer de la bonne analyse fonctionnelle des éléments résiduels $\mathrm{SN}-\mathrm{SN}$.

(56) Le monde social est parsemé de rappels à l'ordre qui ne fonctionnent comme tels que pour les individus prédisposés à les apercevoir, et qui, comme le feu rouge le freinage, déclenchent des dispositions corporelles. Bourdieu (Méditations pascaliennes: 210)

(57) L'aptitude de l'ancêtre à grimper dans les arbres devait le mettre à l'abri, comme aujourd'hui les singes, des grands prédateurs. LE MONDE: 08/02/01:25

En (57), en restituant l'ordre des phrases, en procédant à la copie et au remplacement par les éléments résiduels on obtient (58). Signalons que la simple copie ne suffit pas puisqu'il faut aussi substituer l'ancêtre par des(de-les) singes dans le complément du $\mathrm{N}$ aptitude.

(58) L'aptitude de l'ancêtre à grimper dans les arbres devait le mettre à l'abri des grands prédateurs, comme aujourd'hui l'aptitude des singes à grimper dans les arbres les mettent à l'abri des grands prédateurs.

Les données exposées montrent des disparités telles qu'on ne peut envisager de procédures simples de type copie+effacement. Il faut disposer d'un modèle qui, au minimum, organise sur des plans distincts les informations liées à la constituance et celles relatives à la linéarisation.

\subsection{Avantages d'une approche non dérivationnelle}

Des travaux plus récents ont été menés dans un cadre non dérivationnel, en HPSG en particulier. Ce type de cadre est a priori plus adapté pour rendre compte du phénomène de l'ellipse dans sa globalité. Il permet d'articuler des niveaux de représentations respectivement associés à un type d'information linguistique (linéarité, fonction, sémantique, pragmatique) au moyen de traits. On peut ainsi sélectionner et associer certaines informations, et pas d'autres. Les opérations se font simultanément en prenant la forme de contraintes de bonne formation sur des types d'objets linguistiques. Les représentations ne rendent compte que des réalisations de surface, il est impossible de construire des syntagmes vides.

Dans le champ des travaux sur l'ellipse, nous choisissons de retenir la proposition de Ginzburg \& Sag (2001) qui autorise, sous certaines conditions, l'existence de catégories syntagmatiques fragmentaires. Ceci ouvre la possibilité, contre l'idée même d'une reconstruction syntaxique, qu'une séquence elliptique puisse être intégrée comme une catégorie syntaxique à part entière, bien que très contrainte et présentant une constituance non canonique. La mise en œuvre de fragments est rendue possible grâce à l'approche constructionnelle que le modèle intègre qui brise l'univocité stricte entre les niveaux d'analyse linguistique. On peut dès lors envisager qu'une séquence non phrastique possède une sémantique propositionnelle. 


\subsubsection{L'ellipse, une catégorie fragmentaire}

Un certain nombre d'arguments montrent la nécessité de postuler l'existence de fragments dans la grammaire des langues comme le français. Tout d'abord, considérant le statut du vide que représente l'ellipse, on constate que la séquence manquante n'est pas un vide «sous-catégorisé ", puisqu'elle touche la tête même de la phrase, contrairement aux constituants syntaxiquement contraints à une non-réalisation, comme le constituant manquant d'une dépendance non bornée ou bien le sujet non réalisé des SV infinitifs. En d'autres termes, il s'agit d'un autre type de vide : une absence de structure.

D'autre part, les séquences fragmentaires ne sont pas syntaxiquement autonomes, elles ne peuvent figurer seules (en dehors des réponses, cf. ex. 59b), elles n'apparaissent que dans des constructions particulières et sont soumises aux contraintes propres de ces constructions. En outre, elles ne peuvent être interprétées que dans l'environnement d'une phrase antécédent qui fournit le contexte source.

Enfin, il existe plusieurs environnements syntaxiques où l'on observe l'apparition de fragments; en plus des comparatives et des constructions coordonnées (ou juxtaposées), ils sont aussi possibles dans une phrase enchâssée avec un adverbe polaire (59), et dans les réponses ${ }^{25}$.

(59) a. Paul viendra mais je pense/crois que Marie pas/non.

b. A-Qu'est-ce que tu écoutes ? B- la radio

Par conséquent, la notion de fragment comme catégorie syntagmatique est justifiée.

Nous avançons, à la suite de Ginzburg \& Sag (2001) et Culicover \& Jackendoff (2005), que les séquences elliptiques ne comportent pas de verbe, elles ne résultent pas d'un effacement de matériel. Suivant plus précisément Mouret (2006), on a affaire à une forme syntaxique particulière constituée de fragments, avec une catégorie qui domine exhaustivement le syntagme. Il n'y a pas de tête vide, ce qui se traduit par le fait que les séquences fragmentaires sont des syntagmes sans tête qui donnent lieu à des séquences (ou listes) de $n$ fragments. Abeillé et al. (2008) développent une analyse de ce type pour le gapping en structures coordonnées en français et en roumain. Les fragments ne sont pas considérés comme des constituants de la séquence (ce qui est indépendant du fait qu'ils soient eux mêmes structurés), mais comme une réalisation linéaire (via un trait 'Domaine d'ordre' dédié à l'ordre des mots). Les informations concernant l'interprétation sémantique ne proviennent pas des mécanismes habituels utilisés pour les syntagmes comportant une tête. Ils reposent sur des règles propres au caractère elliptique de la sous-construction qu'ils forment.

A titre d'illustration, nous reproduisons (figure 1) la représentation (simplifiée) de la séquence fragmentaire Marie des pommes dans le contexte de la phrase Jean mange des bananes et Marie des pommes. Le point crucial du traitement réside dans les contraintes d'appariement entre les constituants résiduels - les fragments -, et des constituants de

\footnotetext{
${ }^{25}$ Les réponses fragmentaires constituent le problème linguistique à l'origine duquel Ginzburg \& Sag (2000) ont proposé l'existence de fragments.
} 
la phrase source. Ces contraintes garantissent la bonne formation du calcul sémantique du syntagme fragmentaire. Un trait gérant les informations contextuelles (SALIENTUTTERANCE) permet d'accéder aux constituants de la phrase source (précédente) et une règle de substitution ${ }^{26}$ associée au domaine d'ordre du syntagme (DOMAIN ORDER) permet d'apparier la structure sémantique du verbe capté avec la sémantique des constituants résiduels. Dans un trait dédié à la structure informationnelle les fragments sont repérés comme étant contrastifs, ce qui permet de mettre en parallèle les éléments de la phrase antécédent qui peuvent faire l'objet d'une substitution.

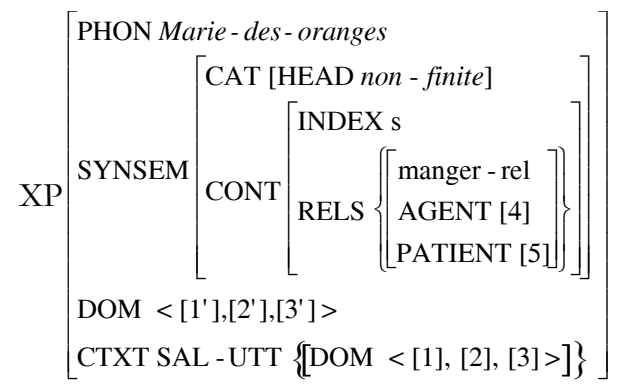

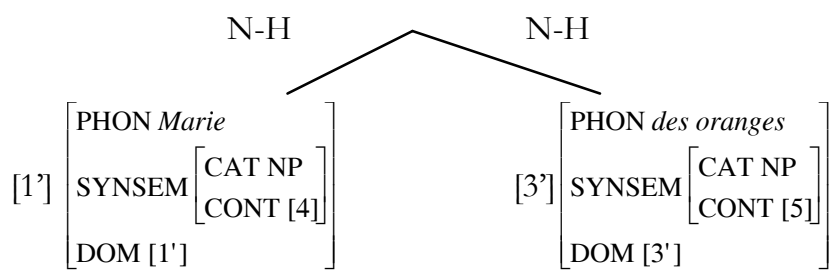

Figure 1 : Structure de traits pour (Jean mange des bananes et) Marie des pommes (in Abeillé et al. 2008).

A priori le traitement proposé par Abeillé et al. (2008) utilise principalement les informations sémantiques pour identifier et vérifier la compatibilité des résiduels avec une relation verbale de la phrase antécédent, ce qui présente l'avantage de ne pas avoir à gérer les disparités formelles (catégorielles). On regrette toutefois que le détail de la règle d'appariement ne soit pas donné. En particulier, que se passe-t-il lorsque le nombre des arguments dans la phrase cible est différent de celui de la phrase source?

Pour l'essentiel, un traitement du même ordre peut être développé pour les séquences fragmentaires des comparatives en comme, modulo certains aménagements pour intégrer les contraintes propres à la construction.

\footnotetext{
${ }^{26}$ La résolution sémantique de l'ellipse proposée dans Abeillé et al. (2008) s'inspire largement des travaux de Sag (1976), puis Gardent (1991), qui, alternativement aux travaux en grammaire générative et transformationnelle, ont proposé un traitement centré sur la résolution sémantique de l'ellipse, par l'intermédiaire d'une duplication de la structure sémantique de la phrase source, puis une règle de substitution permettant d'instancier les arguments résiduels de la phrase cible. De là est dérivée la structure syntaxique. Cette approche a l'avantage d'être moins soumise aux contraintes dues à la copie structurale de la phrase source mais conserve les problèmes liés à un traitement par dérivation (i.e., la nécessité d'ordonner les opérations).
} 


\subsubsection{Remarques sur le traitement de l'ellipse des comparatives en comme}

Le traitement de l'ellipse par fragment peut être intégré de façon cohérente à l'analyse HPSG des comparatives en comme et à celle des relatives sans antécédent. Ceci étant, les comparatives présentent des particularités et des variétés qui rendent le traitement sémantique des fragments plus complexe que ne l'est celui des coordinations de propositions. Plusieurs remarques peuvent être faites à ce sujet.

Une première observation est que les éléments de la phrase source qui permettent l'interprétation de la proposition cible varient selon le type de comparative, ou plus précisément, selon l'élément avec lequel la comparative en comme se combine. On distingue ainsi les comparatives qui se combinent avec un constituant à tête verbale, autrement dit une phrase, dans le cas d'un ajout d'analogie, ou un V/SV dans le cas d'un modifieur ou d'un complément de $\mathrm{V}$, des comparatives prédicatives, qui se combinent avec un adjectif (A/SA) ou avec un nom (N/SN).

Dans le premier cas, à l'instar de ce qui se passe avec les ellipses en coordonnées, la proposition de la séquence fragmentaire se construit en utilisant une relation verbale (assortie de ses arguments si nécessaire) fournie par la phrase source. En ce qui concerne l'ajout d'analogie (60), il n'y a pas d'indication syntaxique du verbe qui doit être utilisé. En revanche, les comparatives modifieurs ou compléments de V (61) utilisent la relation sémantique du V avec lequel elles se combinent. Afin de rendre compte de l'interprétation, nous transcrivons en petits caractères le verbe et les arguments manquants -dont l'adverbe de manière extrait-; mais, il ne s'agit en aucun cas de reconstruction syntaxique.

(60) a. Paul adore la peinture, tout comme sa femme adore la peinture d'ailleurs.

b. Paul adore la peinture, tout comme Paul adore les arts en général.

c. Paul adore la peinture, tout comme sa femme adore les arts en général.

d. Paul a souvent promis d'essayer d'apprendre le latin, comme Marie apromis d'apprendre le grec.

(61) a. Il sonne exactement comme le mot serviette sonne d'une x manière.

b. Celle qui court le long de votre vie comme le feu count d'mne x manière le long d'un cordon Bickford.

c. Ma voisine parle aux gens comme ma voisine parle d'une $x$ manière à son chien.

Le cas des comparatives prédicatives, modifieurs de A/SA ou de N/SN, est remarquable en ce que l'interprétation de la séquence fragmentaire est toujours une structure d'attribution, et qu'aucune relation sémantique verbale propre n'est fournie par la phrase source.

Les comparatives modifieur d'adjectif (ou de SA) présentent deux types de séquences fragmentaires: ou bien, la séquence ne contient qu'un fragment qui correspond (toujours) au sujet (cf. 62a,b,c), ou bien, moins fréquemment, elle contient un fragment sujet et un fragment qui correspond à un attribut (cf. 62d,e). Dans tous les cas, la relation sémantique à reconstituer est une prédication simple ; intuitivement, on utilise le verbe copule pour l'interprétation de la séquence fragmentaire, et ce, indépendamment du verbe utilisé dans la phrase matrice. Lorsque seul un fragment sujet est réalisé, l'attribut manquant correspond à celui utilisé dans la phrase source, le constituant même qui se combine avec l'ajout comparatif. L'adverbe de manière extrait est un modifieur de l'attribut. 
(62) a. Tu sera laide comme les quatorze pêchers capitaux sont $x$ laids.

b. Je l'ai trouvé intelligent comme son frère est $x$ intelligent .

c. Un homme honnête comme toi tu es $x$ honnête ne peut pas mentir.

d. Jean est intelligent comme Paul est $x$ adroit.

e. J'ai trouvé Jean intelligent comme Paul est $x$ adroit.

Les séquences qui sont constituées d'un unique fragment s'interprétant comme un attribut ne relèvent pas de la même analyse. Ou bien, on a affaire à une coordination d'attributs (cf. Mouret \& Desmets, ce numéro), comme en (63a), dans laquelle il n’y a pas de restitution possible de la phrase pleine. Ou bien, on est face à une comparative d'analogie qui utilise l'ensemble de la relation sémantique du verbe de la phrase source (cf.63b)

(63) a. J'ai toujours aimé cet homme intelligent comme bien élevé

b. Je l'ai trouvé intelligent, comme je lai trouvé maladroit

L'interprétation des comparatives fragmentaires modifieurs de $\mathrm{N} / \mathrm{SN}$ ne s'appuie pas non plus sur un verbe réalisé dans la phrase source. Dans tous les cas, il s'agit d'une attribution de propriété simple (avec une copule, s'il fallait reconstruire un verbe) et le $\mathrm{N}$ modifié est utilisé comme un des éléments de la relation prédicative. Soit il fournit l'attribut, et l'adverbe extrait s'interprète alors comme un spécifieur du $\mathrm{N}$ attribut, comme en $(64 \mathrm{a}, \mathrm{b})$; soit le $\mathrm{N}$ modifié fournit le sujet de l'attribution et l'adverbe extrait s'interprète comme l'attribut $(64 c, d)$.

(64) a. Paul adorerait avoir une voiture comme la tienne est une x voiture

b. Marion a de la chance d'avoir une amie comme Agnès est une x amie.

c. J'ai trouvé des pâtisseries comme lespatitsereres sont $x$ chez Mazet.

d. J'ai mangé un ragoût comme les ragoûts sont $x$ chez la cousine Aline.

Une autre particularité des comparatives en comme, et qui peut être problématique pour le traitement tel qu'il est proposé par Abeillé et al. (2008), concerne une possibilité liée à leur fonction : la mobilité.

(65) a. Le monde social est parsemé de rappels à l'ordre qui ne fonctionnent comme tels que pour les individus prédisposés à les apercevoir, et qui, comme le feu rouge le freinage, déclenchent des dispositions corporelles. Bourdieu (Méditations pascaliennes: 210)

b. L'aptitude de l'ancêtre à grimper dans les arbres devait le mettre à l'abri, comme aujourd'hui les singes, des grands prédateurs. LE MONDE : 08/02/01: 25

c. Dans la nôtre [la société], et même si ces dessins danois sont d'une navrante platitude, la caricature est un art ancestral, issu des grotesques, des gargouilles et des masques médiévaux, et un art du peuple, conquis, comme le journal, de haute lutte. (LM - portail web du 11/04/2008)

d. Comme Paul les jours de pluie, Marie se décide à mettre des bottes aujourd'hui.

Les cas d'insertion et d'antéposition illustrés en (65) montrent que le calcul sémantique de la séquence fragmentaire est résolu une fois que l'ensemble de l'énoncé est effectué. Autrement dit, la liste des éléments du domaine de la phrase matrice (du trait SALIENT-UTTERANCE) qui est utilisée pour opérer la substitution ne doit pas être limitée aux éléments précédemment énoncés. L'appariement doit être réalisé au niveau du nœud mère du syntagme tête-ajout(parenthétique) pour avoir accès à l'ensemble des éléments à substituer. 
Enfin, la dernière remarque porte sur la catégorie avec laquelle comme comparatif se combine. Les exemples (70) montrent que la comparative n'accepte pas l'ellipse du sujet ${ }^{27}$.

(66) a. *Jean écoute la radio, comme lit le journal d'ailleurs.

b. *Ces dessins déstabilisent, tout comme désavouent la suprématie du pouvoir religieux d'ailleurs.

On peut en déduire deux choses. D'une part, lorsque la comparative est finie, elle l'est au sens stricte, c'est-à-dire qu'elle comporte un verbe tête conjugué qui réalise obligatoirement un sujet syntaxique ${ }^{28}$ ou clitique (pronom personnel). D'autre part, on confirme le fait que si la phrase est elliptique, elle omet obligatoirement le verbe tête, c'est-à-dire qu'elle doit être strictement sans tête, soit non finie. On pourrait faire l'hypothèse que lexicalement comme comparatif se combine toujours avec un constituant de catégorie Phrase, même lorsque la réalisation de cette dernière est fragmentaire. Le problème est qu'une catégorie phrase possède obligatoirement une tête. Pour Abeillé et al (2008), fragment est une valeur catégorielle (une valeur pour le trait HEAD). Il faut donc spécifier au niveau de la construction comparative que la catégorie avec laquelle comme se combine est soit une phrase (finie) - ce que propose Desmets (2002) -, soit un fragment. Par ailleurs on peut ajouter, suivant une des dimensions d'organisation des syntagmes en HPSG, que les fragments de type gapping sont des clauses, en ce qu'ils possèdent le même type de sémantique que les phrases finies.

\section{Conclusion}

En étudiant les séquences fragmentaires des comparatives en comme, nous avons pu confirmer que l'ellipse est un phénomène trans-constructionnel, qui n'altère pas (ou peu) les propriétés externes des constructions dans lesquelles elle apparaît. Sa présence est un corollaire à la redondance informationnelle, laquelle résulte de parallélismes sémantiques et syntaxiques; cependant le phénomène n'est pas toujours optionnel. L'ellipse est sensible à l'environnement syntaxique dans lequel elle apparaît (ce qui peut expliquer la proximité de l'ellipse des constructions comparatives en comme et celle des comparatives scalaires), ce dont témoigne la comparaison avec l'ellipse en structures coordonnées. Enfin, le phénomène est difficile à modéliser, pour des raisons tant empiriques que théoriques et la réponse théorique la plus adaptée à ce jour semble être une approche par fragment. La phrase elliptique est vue comme une phrase fragmentaire, syntaxiquement non finie, dont la sémantique propositionnelle est reconstruite à partir de la phrase source. Cette approche répond favorablement aux propriétés de l'ellipse des comparatives en comme que nous avons étudiées. La formalisation d'une telle analyse, intégrant les observations du présent article, constituera l'objet d'un travail ultérieur.

\footnotetext{
${ }^{27}$ Une contrainte identique pèse sur les comparatives scalaires: *Jean écoute plus la radio que ne lit le journal; *Ces dessins déstabilisent plus que (ne) désavouent la suprématie du pouvoir religieux.

${ }^{28}$ En outre, le sujet de la phrase se combinant avec comme ne peut être extrait.
} 


\section{RÉFÉRENCES BIBLIOGRAPHIQUES}

ABEILlÉ, A. (à paraitre), "Les phrases elliptiques et fragmentaires », in A. Abeillé, A. Delaveau \& D. Godard (éds.), Grande Grammaire du Français, chp1. (coord.) A. Delaveau. Bayard Presse.

ABEILlÉ, A. (2005), «Les syntagmes conjoints et leurs fonctions syntaxiques », Langages 160, pp. $42-66$.

ABeillé, A., G. Bilbiie, \& F. Mouret (2008), «Gapping in Romance: a fragment analysis », International Conference on Elliptical constructions, LLF \& Université Paris Diderot, Chicago Center, Paris.

ABEILlÉ, A. \& D. Godard (1996), «La complémentation des auxiliaires en francais », Languages 122, pp. 32-61.

ABEILlÉ, A. \& F. Mouret (à paraittre), "Quelques contraintes sur les coordinations elliptiques en francais », Revue de sémantique et de pragmatique.

Amsili, P. \& M. Desmets (2008a), «French Comparative Ellipsis», International Conference on Elliptical constructions, LLF \& Université Paris Diderot, Chicago Center, Paris.

AmsiLi, P. \& M. Desmets (2008b), "Comparatives scalaires parenthétiques», Colloque La scalarité dans tous ses aspects, Gand.

Amsili, P., Desmets, M., Roussarie, L. \& J. Tseng (à paraittre), «Comparatives et consécutives », chp15. (coord.) M. Desmets, in Delaveau, A. et Godard, D. (éds.) Grande Grammaire $d u$ Français. Bayard Presse.

BONAMI, O. \& D. Godard (2003), «Incidentals adjuncts: an overlooked type of adjunction ». HPSG conference.

BonAmi, O., Godard, D. \& B. Kampers-Manhe (2004), «Adverb classification », in F. Corblin et H. de Swart (éds), Handbook of French semantics. Stanford : CSLI Publications, pp. 143-183.

BRESNAN, J. \& J. Grimshaw (1978), «The syntax of free relatives in English », Linguistic Inquiry 9-3, pp. 331-391.

BRESNAN, J. (2000), Lexical-Functional Syntax. Oxford: Blackwell.

Chomsky, N. (1977), On wh-movement. In Peter Culicover, Thomas Wasow, and Adrian Akmajian (eds.), Formal syntax, 71-132. Academic Press: New York.

Culicover, P. \& R. Jackendoff (2005), Simpler Syntax. Oxford University Press.

DESMETS, M. (à paraître), «Les phrases à extraction», in A. Abeillé, A. Delaveau \& D. Godard (éds.), Grande Grammaire du Français, chp1. (coord.) A. Delaveau. Bayard Presse.

Desmets, M. (2002), «French Free Relative Phrases in HPSG», HPSG Conference, Séoul, Corée.

Desmets, M. (2001), Les typages de la phrase en HPSG : le cas des phrases en comme. Thèse de doctorat, Université Paris X- Nanterre.

Desmets, M. \& Roussarie. L. (2001), «French Reportive Comme Clauses : a case of parenthetical adjunction », in D. Flickinger \& A. Kathol (éds.) HPSG Conference, CSLI On-line Publications, pp. 43-62.

GARDENT, C. (1991), Gapping and VP Ellipsis in a Unification-Based Grammar. PhD thesis, University of Edinburgh.

GINZBURG, J. \& I.A. Sag (2001), Interrogative investigations: the form, meaning and use of English interrogatives. Stanford: CSLI Publications. 
Hankamer, J. (1971), Constraints on Deletion in Syntax, New Haven, Yale University Doctoral Dissertation.

HANKAMER \& Sag (1984), "Toward a Theory of Anaphoric Processing», Linguistics and Philosophy, 7, pp. 325-345.

Hudson, R. A., (1976), "Conjunction Reduction, Gapping, and Right-Node Raising ", Language, vol. 52, no3, pp. 535-562.

KeHLer, A. (2002), Coherence, Reference and the Theory of Grammar. CSLI Publications.

KENNEDY, C. \& J. Merchant (2000), «Attributive comparative deletion », Natural Language and Linguistic Theory 18, pp. 89-146.

LE GOFFIC, P. (1991), «Comme, adverbe connecteur intégratif : éléments pour une description », Travaux linguistiques du CERLICO 4, pp. 11-31. Rennes, Presses Universitaires de Rennes 2.

MARANDin, J.-M. (1999), Grammaire de l'incidence. ms. (np). Laboratoire de linguistique formelle, Université Paris 7.

Merchant, J. (2004), «Fragments and Ellipsis », Linguistics and Philosophy 27, pp. 661-738.

Milner, J.-C. (1973), Arguments linguistiques. Paris-Tours, Mame.

Moline, E. (à paraître), «Les propositions d'analogie en comme», in E. Moline \& N. Flaux (éds), Langue Française 157.

Moline, E. (2007), «Détachement, négation et comparaison : un emploi paradoxal de comme ?», in N. Flaux \& D. Stosic (éds), Les constructions détachées : entre langue et discours, Presses Universitaires d'Artois, pp. 129-151.

MOLINE, E. (2001), «Elle ne fait rien comme tout le monde. Les modifieurs adverbiaux de manière en comme », Revue Romane 36-2, Copenhague, pp 171-192.

MOURET, F. (2006), «A phrase structure approach to argument cluster coordination », HPSG Conference, S. Muller (ed), On-line Proceedings, CSLI Publications.

MOURET, F. (2007), Grammaire des constructions coordonnées. Coordinations simples et coordinations à redoublement en français contemporain. Thèse de doctorat, Université Paris 7.

Pullum, G. K. \& A. M. Zwicky (1986), «Phonological resolution of syntactic feature conflict», Language 62, pp. 751-773.

Ross, J. R. (1967), Constraints on variable in syntax, Ph.D., MIT.

SAG, I. A. (1976), Deletion and logical form. PhD thesis. MIT.

TAssin, G. (1998), L'ellipse dans la construction à Gapping. Mémoire de DEA, Université Paris X, Nanterre.

Tellier, C. (1991), Licensing Theory and French Parasitic Gaps. Kluwer Academic.

WASOw, T. (1972), Anaphoric Relations in English, Unpublished Doctoral Dissertation, MIT, Cambridge, Mass.

WiLliams, E. (1977), « Discourse and logical form », Linguistic Inquiry, 8(1), pp. 101-139.

ZRIBI-HERTZ, A. (1986), Relations anaphoriques en français : esquisse d'une grammaire générative raisonnée de la réflexivité et l'ellipse structurale, Thèse de Doctorat d'Etat, Université Paris 8. 